\title{
New motor control assessment techniques for evaluating individuals with severe handicaps: A case study*
}

\author{
NATHAN J. RUDIN; L. DONALD GILMORE; SERGE H. ROY; CARLO J. DE LUCA \\ Boston University NeuroMuscular Research Center, 620 Commonwealth Avenue, Boston, Massachusetts 02215
}

\begin{abstract}
Prescription of assistive devices for motorhandicapped individuals requires assessment of their motor capabilities. When patients' motor deficits are particularly severe, wide individual differences in the location and type of abnormalities complicate the assessment process. The precision of assessment has been greatly increased in recent years by the use of quantitative, computer-aided motion analysis, which facilitates statistical examination and comparison with normal individuals.

This paper discusses a case study wherein a 24-yearold male nonvocal cerebral palsy patient was assessed for his ability to operate assistive communication devices. Three computer-aided measurement protocols were employed to evaluate the patient and two controls: 1) performance using the patient's existing communication aid was evaluated in terms of rate and accuracy of communication using standardized spelling and response time tasks; 2) volitional myoelectric activity was surveyed to identify possible myoelectric control sites for communication aid operation; 3 ) a study of head position and its time derivatives was conducted to explore the feasibility of proportional control of a communication aid. Comparison of handicapped and control subject data indicated that, despite several characteristic motor control deficits, the handicapped subject was capable of proportional control of lateral head rotation and binary control of frontalis myoelectric signals. These movements could be used to operate a proportionally-controlled, direct-selection communication aid that could substan-
\end{abstract}

\footnotetext{
*This research was supported in part by a Boston University Graduate Fellowship, Liberty Mutual Insurance Company, and the Veterans Administration Rehabilitation Research and Development Service.
}

tially increase the subject's communication rate. Work in progress includes the expansion of the handicapped and unimpaired subject databases and further development of the techniques discussed here to include threedimensional motion analysis and objective measurement of muscle fatigue.

DOI $10.1682 / J R R D .1987 .07 .0057$

\section{INTRODUCTION}

Numerous situations arise in the clinical treatment of motor-handicapped individuals where their motor capabilities must be assessed. Prescribing wheelchairs, communication devices, and other aids requires measurement of the person's ability to move body segments when performing various tasks. Clinicians use the resulting data to determine the degree of the patient's disability and the appropriate intervention. Unfortunately, when dealing with patients having severe motor disabilities, such an assessment can be extremely difficult to perform. In many cases, before a person's volitional motor control can be assessed, the clinician must first identify the (often few) sites where such control exists. This task is complicated by the widely varying physical and cognitive abilities of the severely motor-handicapped and the fact that their movement abnormalities may mask the presence of usable voluntary control. For example, spastic flailing of the arms may draw attention away from voluntary control of the digits (4). Should the clinician fail to detect all 
Journal of Rehabilitation Research and Development Vol. 24 No. 3 Summer 1987

usable control sites, or fail to take into consideration the patient's cognitive capacities, the device prescribed may not fully utilize the patient's potential to interact with his/her environment (14).

Until recent years, most motor assessment of severely handicapped individuals was carried out using relatively simple clinical techniques such as the use of protractors to evaluate range of motion (3). Today, however, the precision of motor assessment has been greatly increased by the ready availability of computers. Increasing numbers of researchers $(2,9,10,17)$ are turning to computer-based motion analysis to supplement the standard clinical measures. The most apparent virtue of this new technology is its precise quantitative measurement. Quantitative descriptions of motor performance facilitate comparison with normal individuals and, given a database of specifications for various assistive devices, could greatly simplify the patient/ device matching process by providing a clear summary of the patient's motor resources. [Goodenough-Trepagnier, Rosen and their colleagues (6) are currently assembling such a database.]

This paper describes the development and implementation of several techniques using computerbased motion analysis for motor assessment of the severely handicapped. Included are methods for the assessment of spatial displacement of body segments and its time derivatives. Besides providing highprecision quantitative data, these techniques have an additional advantage: whereas many previous methods assess the performance of discrete, unrelated movements, the new techniques can assess motion during dynamic tasks that involve the execution of a series of related movements over time $(7,17)$. The use of assistive devices is most often a continuous process involving long "chains" of manipulations and corrections, and dynamic tasks approximate this process far more closely than do unconnected movements. Also discussed is a method for the collection and interpretation of myoelectric (ME) signals, a potentially useful source of device control which remains largely untapped.

All of the techniques use relatively simple instrumentation and promise to be applicable to the assessment of a wide variety of movements. To test their effectiveness, the techniques were applied to the prescription of assistive communication devices, a typical clinical task requiring detailed motor assessment. A severely handicapped nonvocal cere- bral palsy patient and two nonhandicapped volunteers served as subjects in a case study.

The goal of this study was the generation of a list of the handicapped patient's best possible sites for communication device control, with displacement and myoelectric parameters provided for each site. These parameters were compared to those of the normal subjects in order to determine the degree of the patient's motor impairment. Finally, the data were used to suggest the types of communication devices that the patient could best operate.

\section{PROCEDURE}

The examination was performed on a 24-year-old male handicapped subject having severe cerebral palsy with mixed spastic and athetoid components. Functionally a quadriplegic, with uncontrollable spasticity and rigidity in most muscles, the subject is completely nonvocal because of severe dysarthria. According to tests administered by his physicians, the subject's intelligence, perceptual abilities and written language skills are normal for his age level.

For the purpose of comparison, two students with no neurological impairment participated as control subjects: a 21-year-old female and a 22-year-old male.

Each subject was evaluated according to the procedure described below to determine the extent of his/her voluntary motor capabilities. As all three subjects possessed unimpaired cognitive and perceptual abilities, these variables were not assessed during this study. The assessment procedure used is an extension of the clinical assessment normally performed during communication device prescription, with additional features added to more extensively quantify the subject's motor control. (Figure 1) As in the normal assessment, medical records and other data gathered previous to the test, plus interview results, are combined to generate a list of potential control sites. These sites are then evaluated using three separate protocols. First, performance using the handicapped subject's current communication device is evaluated to observe each subject's control of and communication rate using the relevant control sites. The second and third protocols allow detailed observation of motor control through evaluation of myoelectric activity and displacement of body segments. In both protocols, the handicapped 
Interview and Compilation of Previous observations

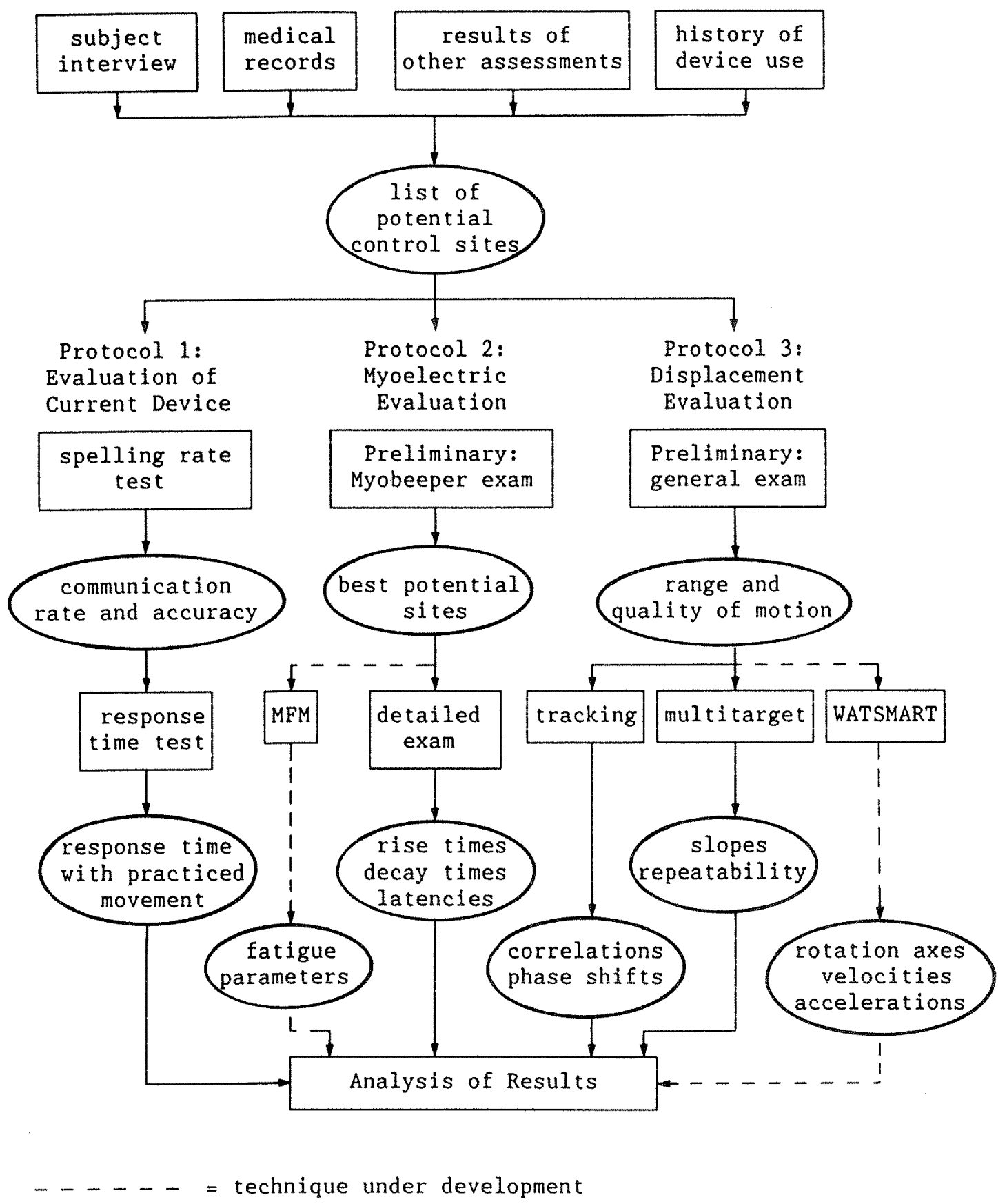

Figure 1.

Flow diagram of motor control assessment procedure illustrating the various tests (in rectangles) and their results (in ovals).

subject's body is systematically scanned to eliminate unusable sites, narrowing the list of possible control sites to those having the greatest potential for use. The remaining sites are then subjected to in-depth quantitative assessment. The result is a focused list of preferred control sites, with quantitative parameters provided for each site, ready for analysis and comparison with data collected from other subjects. 
Journal of Rehabilitation Research and Development Vol. 24 No. 3 Summer 1987

\section{Interview and Compilation of Previous Observations}

An interview was conducted with the handicapped subject to establish his history of motor assessment and communication device use and his needs regarding a communication device. Copies of the subject's medical records were obtained and searched for information on his motor capabilities, as well as the results of any previous communication evaluations. The data from these sources were compiled to produce a list of the subject's most probable potential control sites, as indicated in the oval near the top of Figure 1. The most probable sites were head movement, with control better in side-to-side horizontal rotation; hip flexion from a sitting position; activation of facial muscles; and controlled direction of gaze.

\section{Protocol 1: Evaluation of Currently Used Device}

This evaluation was conducted to ascertain the handicapped subject's rate and accuracy of communication while performing a long-practiced communication task. The handicapped subject's chief method of communication was through the UNICOM electronic communicator (18), which projects a matrix of characters on a video display. The subject used the device in row-column scanning mode, operating it by moving his head to actuate a switch mounted near the right cheek. (Figure 2) Two actuations were required to select a single letter or number. Performance of this practiced and apparently well-controlled movement was evaluated relative to normal subjects. To accomplish this evaluation, a controlled situation was created in the form of a spelling rate test wherein standard sentences were presented to each subject for duplication using UNICOM. A response time test, also using the UNICOM switch, was administered to study the maximum rate of UNICOM switch actuation independent of the spelling task.

Spelling Rate Test: Each subject was seated comfortably in a chair on which the UNICOM control switch had been mounted and positioned to rest approximately $10 \mathrm{~cm}$ to the right of the right cheek. The switch was connected to UNICOM and the chair positioned before UNICOM as in Figure 2 . UNICOM was set at the scan rate normally used by the handicapped subject (approx $0.8 \mathrm{scans} / \mathrm{s}$ ). Each subject was sequentially presented with three test sentences printed in 2-cm-high letters on white cards held $2 \mathrm{~m}$ from the subject's face. Each subject was instructed to duplicate the sentences as rapidly as possible using UNICOM. Time for sentence completion was recorded, and mean correct selection rate and number of errors were calculated.

Response Time Test: The response time test measures the time required for a subject to execute a specified movement after a signaling stimulus is presented. The response time to an audible stimulus involves two normally inseparable components: reception and processing of the incoming stimulus, and initiation and execution of the required response. Given that previous measures of the handicapped subject's neurological function and intelligence were within the normal range, it can be assumed that any differences observed between his response times and those of normal subjects are reflective of motor rather than information-processing deficits.

Subjects were seated in the chair as in the spelling test, and the switch used for UNICOM was connected to a response-time testing unit. The evaluator controlled a switch that produced a loud stimulus tone. Subjects were instructed to actuate the UNICOM switch as quickly as possible after hearing the tone. Twelve stimulus trials with random intertrial intervals ( 2 to $5 \mathrm{~s}$ ) were presented to each subject. Stimulus and response signals were stored on an FM tape recorder, and response time for each trial was calculated as the interval between stimulus presentation and subject response. Mean response time was calculated. Control and handicapped subject data were compared and tested for statistical significance.

Protocols 2 and 3 are designed to allow particularly detailed observation of motor control. Myoelectric and displacement evaluations were conducted to discover device control possibilities and to increase general understanding of the handicapped subject's motor impairments. Displacement is the most obvious consequence of neuromuscular activity and is the parameter principally observed during most motor assessments. However, the addition of a myoelectric assessment protocol provides a means of observing the control of individual muscle groups during gross displacement of body segments. Each protocol provides a quantitative indication of the subject's motor control abilities, but does not necessarily suggest strategies for control of specific assistive devices. 


\section{Protocol 2: Myoelectric Evaluation}

Preliminary assessment of myoelectric activity was conducted using the Myobeeper (12) (Figure 3), a portable myofeedback device using an active surface electrode (5) (Figure 4). This electrode, which requires no paste or gel, is ideally suited for easy and accurate $\mathrm{ME}$ signal observation. Detected signals were displayed on a meter and as an audio tone.

Each subject was asked to perform a series of muscle contractions (19). The muscles involved were divided into four groups according to location: head and neck, upper limbs and digits, lower limbs, and torso. Before beginning each contraction, the surface electrode was placed over the target muscle. The subject was instructed to begin the specified contraction on the evaluator's command and sustain it until asked to stop. The Myobeeper gain was adjusted for each contraction so that the signal produced a full sweep of the meter, and gain settings were recorded. The latency between the evaluator's commands and the actual beginning and ending of each contraction was measured using a stopwatch. Each task was performed three times. Those muscle sites of the handicapped subject at which gains, durations, and response latencies approximated the

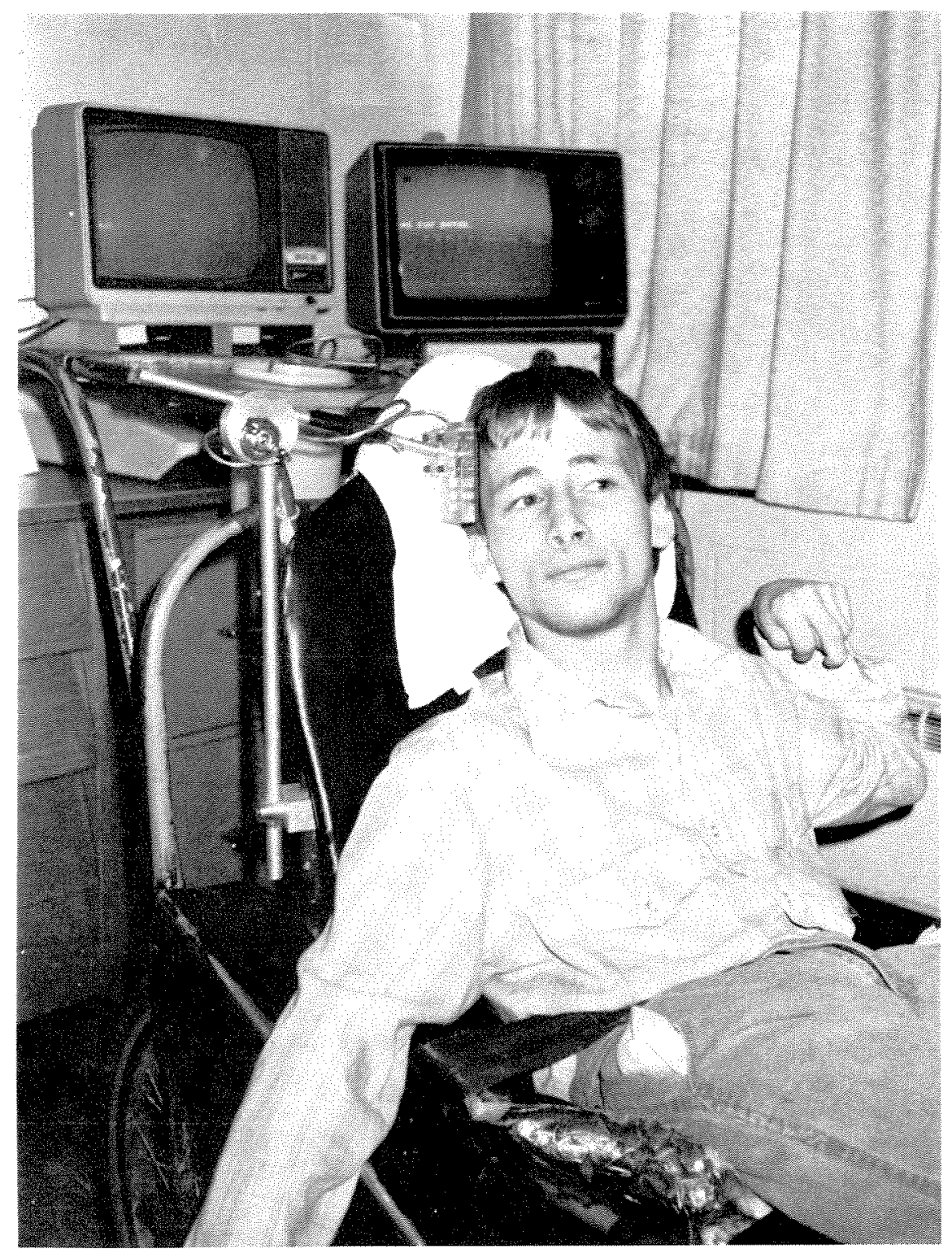

Figure 2.

Handicapped subject using UNICOM. Control switch is mounted to the right of the head. 
normal were identified as preferred for in-depth evaluation. All sites so identified proved to be located on the head or neck.

During in-depth evaluation, surface electrodes were placed over eight potential control sites: the left and right frontalis, masseter, buccinator, and sternocleidomastoid muscles. (Figure 4, top) The subjects were asked to perform a series of contractions (19) involving these muscles, both separately and in combination. Each contraction was performed three times. All ME signals were amplified by 2000 , bandpass filtered from 20 to $550 \mathrm{~Hz}$, and stored using an FM tape recorder. The rise times (time from 10 to 90 percent maximum amplitude), decay times (time from 90 to 10 percent maximum amplitude) and total durations of the resulting envelopes were later measured using a digital storage oscilloscope. Latency of response to the evaluator's commands was also recorded.

\section{Protocol 3: Displacement Evaluation}

As in the preliminary myoelectric evaluation, the body segments of interest were divided into groups involving head/neck, upper limbs and digits, lower limbs, and torso. The subject was seated and asked to displace these body segments relative to appropriately chosen stable body reference points; for example, movement of forearm relative to upper arm (19). Each movement was evaluated in terms of range of displacement of the specified part (in degrees, measured using a protractor) and quality of movement relative to the reference point (measured on a four-point scale ranging from 1 [smooth] to 4 [severe spasticity]). Measurement of movement accuracy was deferred until the later phases of the evaluation. Each movement was performed three times. Sites with movement quality ratings of 1 or 2 were targeted as preferred for in-depth evaluation. Sites having lower ranges of displacement than normal were still identified as preferred, provided that movement of quality 1 or 2 was present within the limited range. As mean values for range and resolution of motion were known for normal individuals (13) the controls were not evaluated at this stage.

The subject's head/neck region was the location

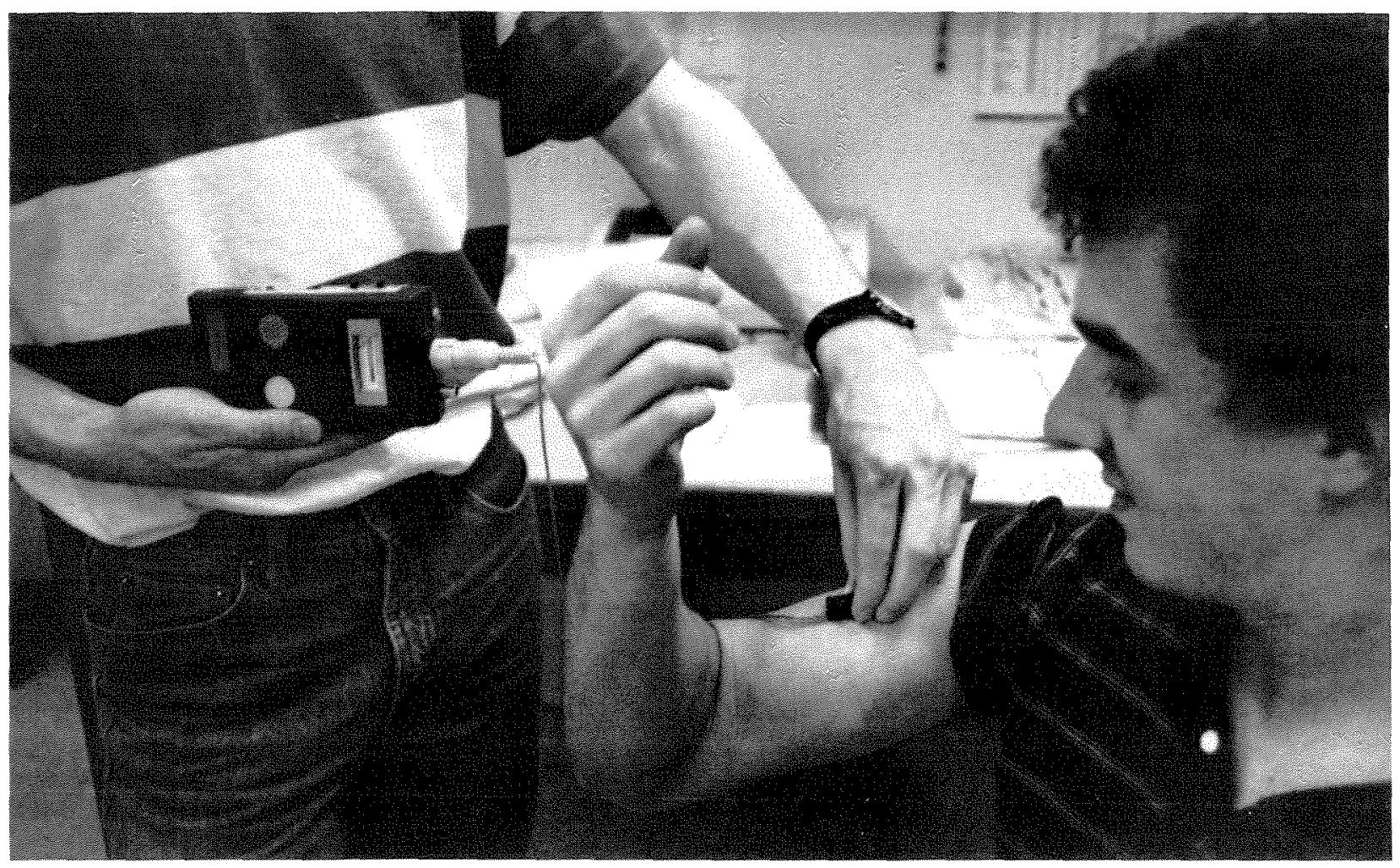

Figure 3.

Typical Myobeeper test situation. The electrode is held or taped to the skin over the muscle of interest. 
of the one site classified as preferred for in-depth evaluation. Movement quality and range of displacement as compared to known normal values were progressively less in the upper limbs and digits, followed by the lower limbs and torso.

The preliminary assessment tasks revealed that head rotation was the preferred control modality for the handicapped subject. Two tasks were used to further evaluate head control: a pursuit tracking task, which measures a subject's ability to trace a defined signal while observing feedback of performance, and a multitarget acquisition task, which examines velocity, resolution, and other parameters related to proportional control. Both the handicapped and control subjects were evaluated.

Tracking Evaluation: Tracking tasks are considered to be an effective means of accurately and objectively assessing integrated sensory-motor function (10). Tracking ability is a sensitive measure of proportionally controlled movement, possibly the most effective modality for communication device control (4). Tracking tasks would therefore seem ideal for application to communication device prescription, but have rarely been used for that purpose. A computerized task that effectively fills this function is described below. In a pursuit tracking task, subjects are presented with both a reference input and a response signal. The subject's task is to make the response signal follow the reference input as closely as possible (7).

An electromagnetic motion transducer (Figure 5) was constructed to measure left and right head rotation. The device utilizes a principle similar to Remmel's (15) search-coil technique for monitoring

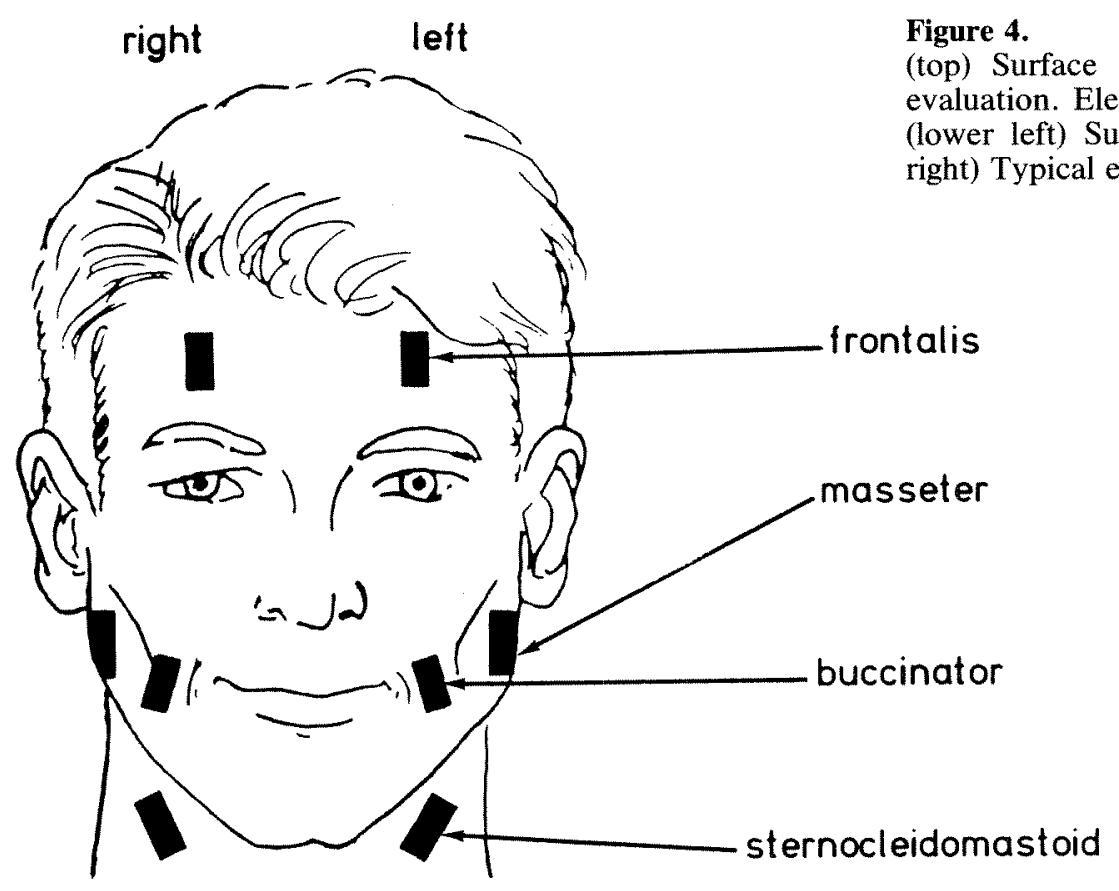

Figure 4.

(top) Surface electrode placement for focused myoelectric odes were held in place using first-aid tape. (lower left) Surface electrode, front and side views. (lower right) Typical electrode recording configuration.
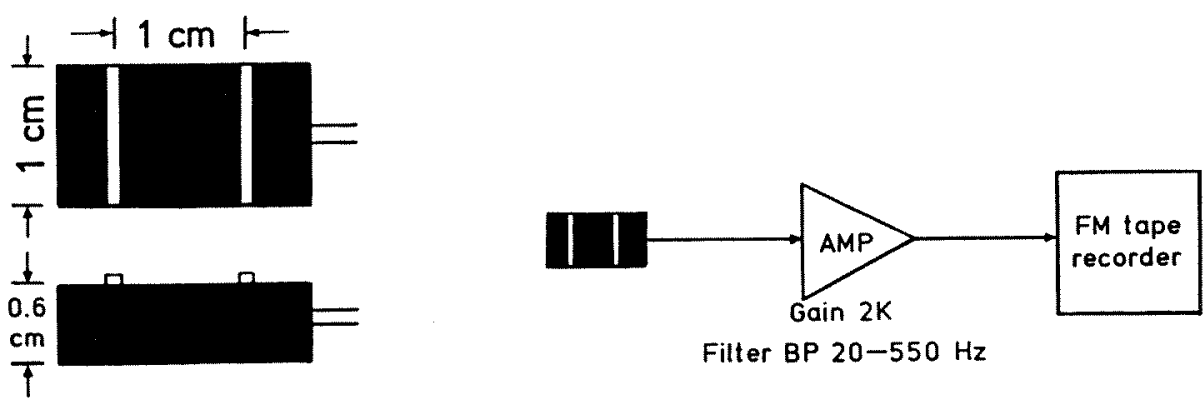

Filler BP $20-550 \mathrm{~Hz}$ 
eye movement. Here, the subject's head is centered between a pair of transmission coils connected to a sine-wave generator. A small coil attached to the head by a plastic headband detects the electromagnetic field produced by the transmission coils. Turning the head changes the amplitude and phase of the voltage in the receiving coil as its angle changes relative to the electromagnetic field. The receiving coil voltage is translated into a direct current voltage proportional to head rotation, making the user's head the functional equivalent of a single-axis "joystick" control.

The tracking task was presented to the subjects using an IBM Personal Computer with an analogto-digital (A/D) data acquisition system. (Figure 6) The reference input was produced by a function generator; the subject's head rotation, detected and processed through the motion transducer, provided the response signal. Both signals were fed into the computer through $\mathrm{A} / \mathrm{D}$ inputs, digitized at a $50-\mathrm{Hz}$ sampling rate, and processed to horizontally move separate cursors displayed on the monitor. The task control program was written in compiled IBM PC BASIC.

The handicapped and control subjects were evaluated. Each subject was seated $1 \mathrm{~m}$ from the IBM PC monitor, positioned in the motion transducer apparatus, and presented with a video display of two cursors positioned one above the other. A white cursor represented the reference input; a yellow cursor plotted the response signal. Subjects were asked to use head rotation to make the yellow cursor follow the white cursor as closely as possible.

Sine and square wave reference inputs were used to examine different aspects of the subjects' motor control. Sine wave tracking involves gradual, nonballistic changes in position, whereas square wave tracking requires rapid motion from one amplitude extreme to the other. Preliminary trials with the handicapped subject showed marked deterioration

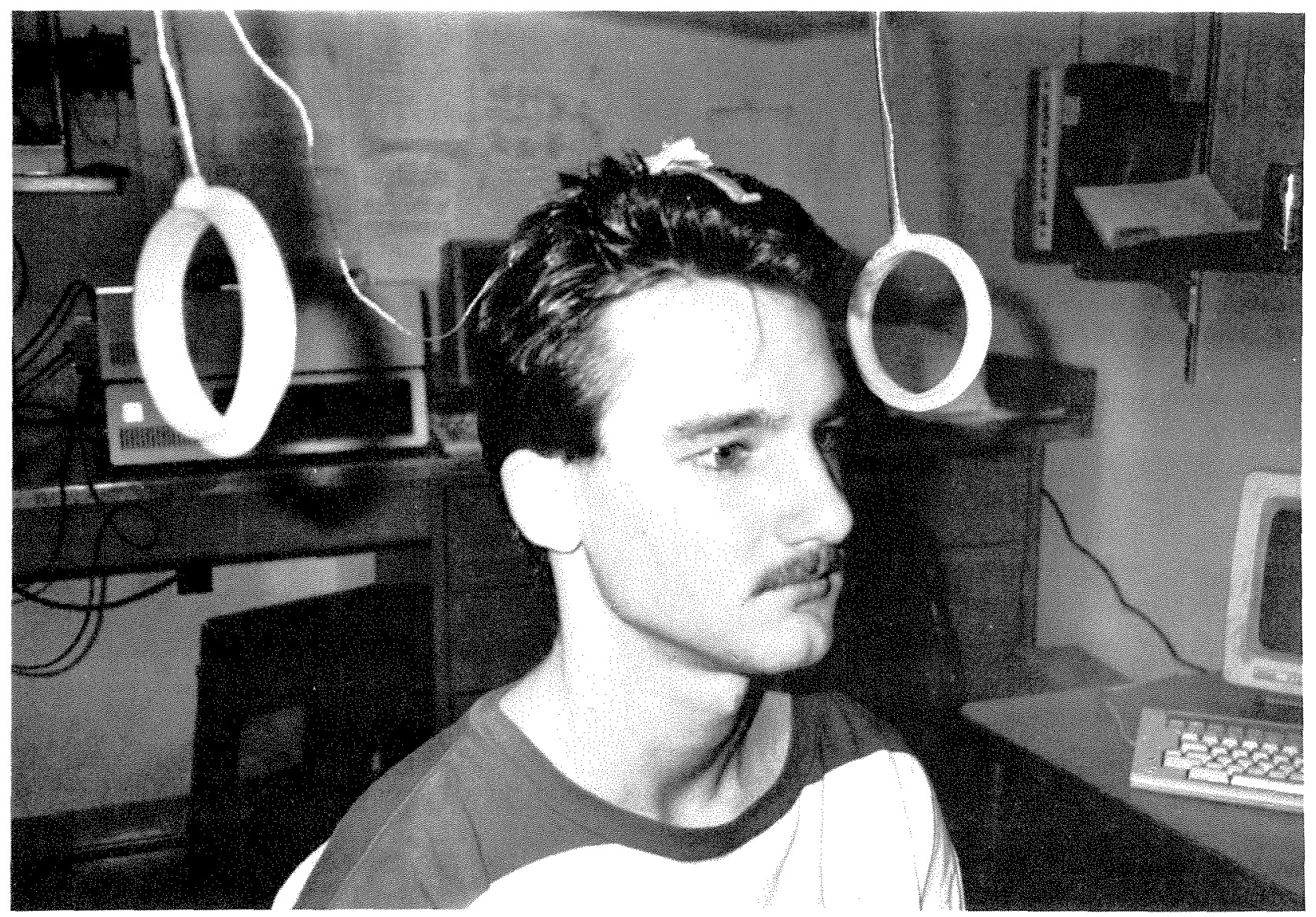

Figure 5.

Electromagnetic motion transducer in use during a tracking task. White rings on either side of head are transmission coils; receiving coil rests atop the head. 
of tracking ability at reference input frequencies above $0.10 \mathrm{~Hz}$; therefore, 0.05 and $0.10 \mathrm{~Hz}$ were selected as test frequencies.

Three different reference inputs were presented, appearing as horizontal excursions of the white cursor across the screen. A one-minute rest period was provided between tasks. The first task lasted 4 min to allow for acclimation; each subsequent task was 2 min long. The reference inputs presented were: $0.05 \mathrm{~Hz}$ sine wave; $0.10 \mathrm{~Hz}$ sine wave; 0.10 $\mathrm{Hz}$ square wave. Maximum amplitude of all three signals was full excursion across the video monitor, which corresponds to a head rotation of 13.2 degrees left to right for the subject and represents a normal range of motion for operation of a head-controlled communication device.

The task data were transferred from the IBM PC to a VAX 750 computer. To determine the phase lag between the reference and response signals, the data were broken into individual cycles. Five cycles were averaged and the mean signal cross-correlated over a time shift of $3 \mathrm{~s}$ in $0.02 \mathrm{~s}$ steps, using a program written in FORTRAN.

Multitarget Acquisition Task: This task specifically assesses the ability to select prescribed targets during proportional head rotation while observing visual feedback of performance. The handicapped subject's movements during target selection can be examined to identify any characteristic signature or template of response. The procedure is a modification of the "Type I" motor assessment described by Goodenough-Trepagnier and Rosen (6) and per- formed on normal subjects by Jandura (9), wherein subjects are instructed to alternately "tap" physical or video targets of various sizes and intertarget distances. For the scope of this study, fixed sizes and distances were chosen for the best observed acquisition performance during preliminary trials. Performance was evaluated based upon the number of successful target acquisitions during a prescribed task.

The electromagnetic motion transducer for head rotation and IBM PC were used as in the tracking evaluation. The transducer control voltage was fed into the $\mathrm{PC}$, digitized at a $20-\mathrm{Hz}$ sampling rate, and used to horizontally move a single cursor between a set of $2.5 \mathrm{~cm}$-wide rectangular targets displayed on the video monitor. (Figure 7) Subjects were seated as in the tracking evaluation. Trial duration was 5 minutes. For the first half of each trial, subjects were instructed to select the targets in patterns specified by the evaluator. For the remainder of the trial, subjects were asked to select whatever targets they wished at the fastest possible speed. A successful selection required that the cursor remain within the target zone for $0.5 \mathrm{~s}$. A beep tone was sounded following completion of this requirement. Two trials were administered to the handicapped subject; but because of the control subjects' faster learning time and more rapid movement, only one trial was administered to each of them.

The data were analyzed using a BASIC program that calculated 2-s time windows representing common target-to-target paths. Each window repre-

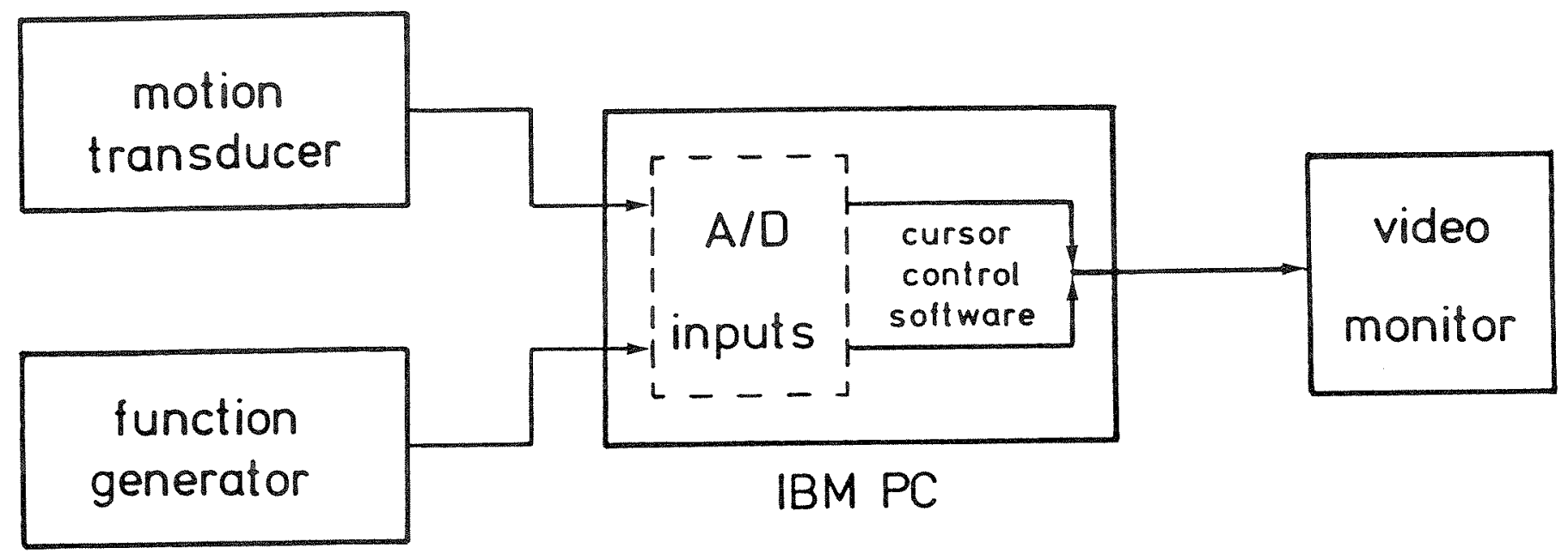

Figure 6.

System diagram for pursuit tracking task. Similar setup (minus function generator) is used for multitarget acquisition task. 
sented the average of from 8 to 20 target-selection events. Averages of absolute position, slope at target entry and exit, time to reach zero slope within target, and total time within target were calculated for each window. Mean acquisition rate in seconds per selection was also calculated. Control and handicapped subject data were compared and tested for statistical significance.
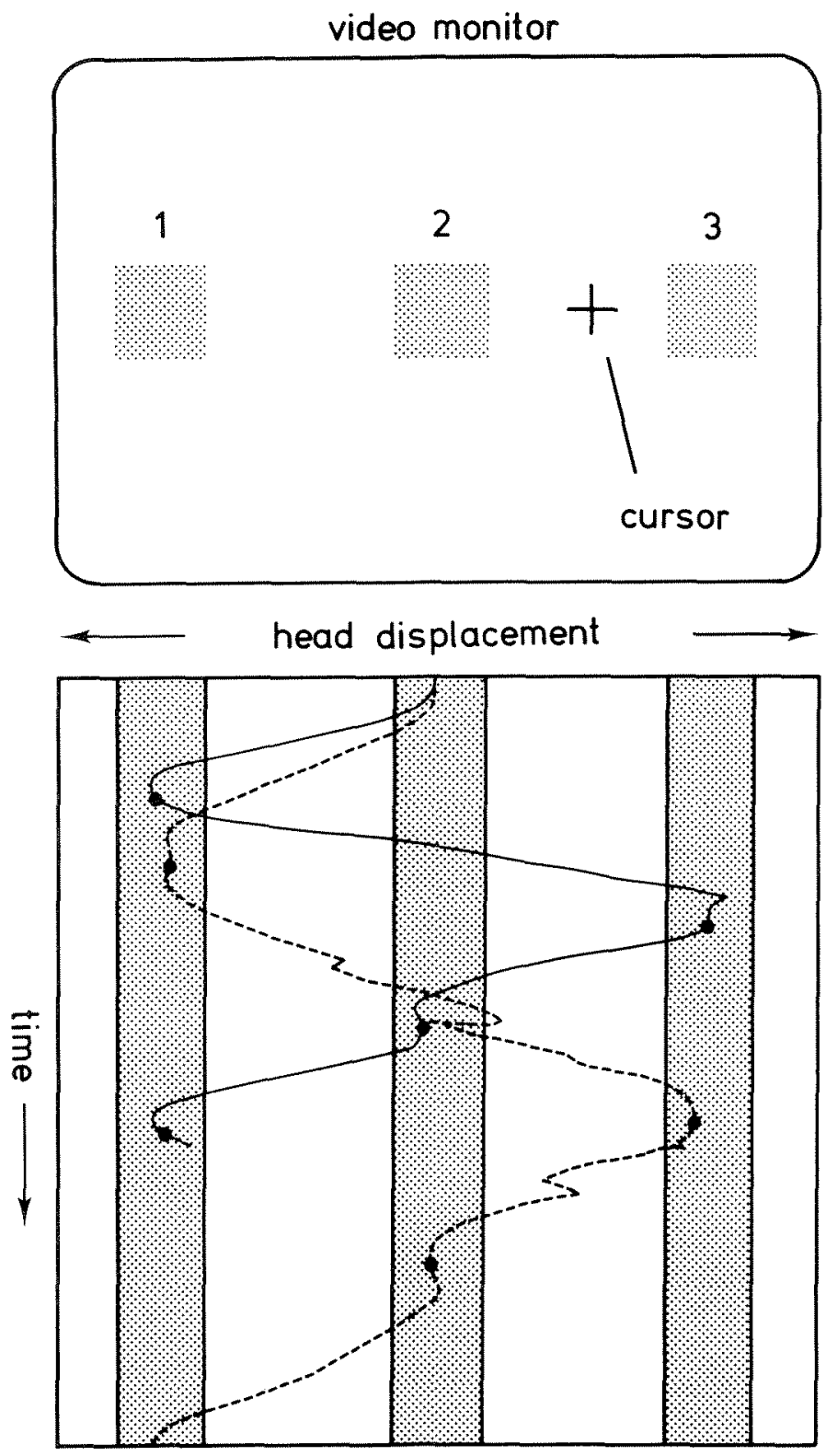

Figure 7.

Multitarget acquisition task with simulated plot of control versus handicapped performance. Cursor moves horizontally between the three targets. Head position is sampled at $20 \mathrm{~Hz}$. Cursor must remain within target $0.5 \mathrm{~s}$ for a successful selection (indicated by black dots).

\section{RESULTS}

\section{Protocol 1: Evaluation of Currently Used Device}

The results of the spelling rate and response time tests are summarized in Table $\mathbf{1}$. In the spelling rate test, the handicapped subject demonstrated a lower communication rate and a higher percentage of incorrect selections than the control subjects. In the response time test, the handicapped subject's mean response time to the audible stimulus was significantly higher than control levels. No significant difference was observed between control subjects in either test. The latter finding suggests that the observed difference in UNICOM communication rate is a result of the handicapped subject's motor limitations rather than information processing deficits.

\section{Protocol 2: Myoelectric Evaluation}

In the preliminary evaluations, the handicapped subject generated signals with durations and response times of ME envelopes approximating control subject levels in the buccinators (by smiling), the masseters (by clenching the teeth), the sternocleidomastoids (by moving the head), and the frontalis (by flexing the forehead). These sites were therefore identified as preferred.

A characteristic deficit pattern was observed at most other sites tested. The handicapped subject, like the controls, was able to initiate the prescribed tasks voluntarily within 1 second of the evaluator's command, and signal gains ranged from 75 to 100 percent of control levels. However, the subject was unable to terminate the task on command; instead, the muscles involved would produce a sustained signal from the Myobeeper at or above control threshold levels. When the task involved a limb, spastic oscillations of the limb and corresponding fluctuations in the sustained signal envelope were observed during the subject's attempt to relax the tetanized muscles. This pattern was characteristic of most of the subject's movements below the neck, with the exception of a few muscles such as the gastrocnemius which were completely rigid at all times. Control subjects demonstrated no rigidity or spasms and terminated all of the specified ME signals within 1 second of the stop command.

Upon detailed examination of the sites classified as promising, it was discovered that these sites, 
Table 1

Results of spelling rate and response time tests. $* *=$ significant, $\mathrm{p}<.05$.

\begin{tabular}{|l|c|c|}
\hline \multicolumn{3}{|c|}{ Spelling Rate Test } \\
\hline subject & $\begin{array}{c}\text { mean correct } \\
\text { selection rate* } \\
\text { (char/min) }\end{array}$ & $\begin{array}{c}\text { errors } \\
\text { made }\end{array}$ \\
\hline handicapped & 4.7 & 11 \\
control 1 & 7.5 & 3 \\
control 2 & 7.3 & 4 \\
\hline
\end{tabular}

$* 50$ correct selections required to complete task

\begin{tabular}{|c|c|c|}
\hline \multicolumn{3}{|c|}{ Response Time Test } \\
\hline subject & $\begin{array}{c}\text { mean response } \\
\text { time }(\mathrm{sec})\end{array}$ & statistical significance \\
\hline $\begin{array}{l}\text { handicapped } \\
\text { control } 1 \\
\text { control } 2\end{array}$ & $\begin{array}{l}0.738 \pm 0.285 \\
0.301 \pm 0.072 \\
0.283 \pm 0.077\end{array}$ & $\begin{array}{l}* * \text { handicapped } \times \text { control } 1: \\
t=5.15, p=0.00 \\
* * \text { handicapped } \times \text { control } 2: \\
t=5.34, p=0.00 \\
\text { control } 1 \times \text { control } 2: \\
t=0.59, \mathrm{NS}\end{array}$ \\
\hline
\end{tabular}

$* *$ significant

with one exception, also demonstrated the fastacquisition, slow-termination pattern observed at most of the other sites. (Figure 8) The two control subjects' parameters were similar across all items tested. For all three subjects, initiation response latencies at all sites tested were less than $0.5 \mathrm{~s}$. Control rise times ranged from $0.3 \mathrm{~s}$ at the frontalis to $1.0 \mathrm{~s}$ at the sternocleidomastoids; the handicapped subject's times were slightly longer, ranging from $0.5 \mathrm{~s}$ at the frontalis to $1.6 \mathrm{~s}$ at the sternocleidomastoids. The handicapped subject's maximum peak envelope amplitudes were 90 to 100 percent of normal at the buccinators and masseters, 80 percent of normal at the frontalis, and 50 percent of normal at the sternocleidomastoids.

Termination response latency for the control subjects was within $0.5 \mathrm{~s}$, and peak amplitude envelope decay times ranged from $0.4 \mathrm{~s}$ at the frontalis to $2.2 \mathrm{~s}$ at the buccinators. Though Myobeeper examination had tentatively identified them as preferred sites, decay times of the handicapped subject's sternocleidomastoid, buccinator and masseter envelopes were up to seven times longer than normal, with a mean of $9.5 \mathrm{~s}$ and a maximum of $11.5 \mathrm{~s}$ decay time at the buccinators. Response latencies were also increased, ranging from 0.5 to $1.5 \mathrm{~s}$.
The normal subjects were able to proportionally control the amplitude of signals from the frontalis muscle, generating signals at 40,75 , and 100 percent of maximum amplitude. The handicapped subject could produce frontalis signals only at or near the 100 percent amplitude level. However, he was able to produce signals with amplitude, rise time, decay time (mean $0.7 \mathrm{~s})$ and response latencies $(0.5 \mathrm{~s}$ or less) in the control range. The frontalis was therefore clearly identified as the preferred potential myoelectric site for communication device control.

\section{Protocol 3: Displacement Evaluation}

As noted in Protocol 2, most of the handicapped subject's voluntary movements were impaired by uncontrollable spasms or rigidity after successful initiation of the movement. Ranges of motion of the arms and fingers approximated normal, but maximum range was reached as a result of spastic rather than volitional movements. Because of constant rigidity in the legs, hip flexion in a sitting position (a movement described as potentially useful by the subject during the initial interview) was limited to 10 degrees of motion as opposed to 40-45 degree for normals (13), and flexion and extension at the knee were completely absent.

The one preferred movement was head rotation, which was already being used to a limited extent to control the subject's communication aid. Proportional control appeared to be present over a range of \pm 35 degrees from center (a typical normal value is 45 degrees) (13). The subject was able to rotate his head to the left and right in 5-degree increments, initiating and terminating movement on command. In contrast, head movements up and down (nodding) were less well-controlled. The subject could raise the head on command but could not lower it in a controlled fashion, instead simply allowing it to fall forward. He was unable to make incremental vertical head movements.

Tracking Evaluation: Correlation coefficients and phase shifts for all subjects are presented in Table 2. All three subjects used head rotation to closely approximate the reference waveforms, with correlation coefficients of 0.63 or better in all cases. Coefficients for the handicapped subject are all lower than those for the controls, but characteristic differences were observed between the controls as well, with one control's coefficients approaching 
Journal of Rehabilitation Research and Development Vol. 24 No. 3 Summer 1987

1.00 while the other control's values were closer to those of the handicapped subject. For all three subjects, correlation decreased as the waveform frequency increased.

The phase lag figures reveal substantial differences in pursuit tracking ability between the handicapped and control subjects. During the sine-wave tracking tasks, both control subjects tended to lag behind the reference cursor. (See Table 2) The handicapped subject, however, shows a negative phase shift. As illustrated in Figure 9 (top), the subject cannot track smoothly at the extremes of the reference signal, but lags behind it while tracking to the right and leads it while tracking to the left, as illustrated by the sinusoidal error-versus-time curve. Lead exceeds lag, resulting in a negative overall phase shift. As wave frequency increases, lag increases (for all three subjects), and the phase shift becomes increas-

\section{LEFT BUCCINATOR}

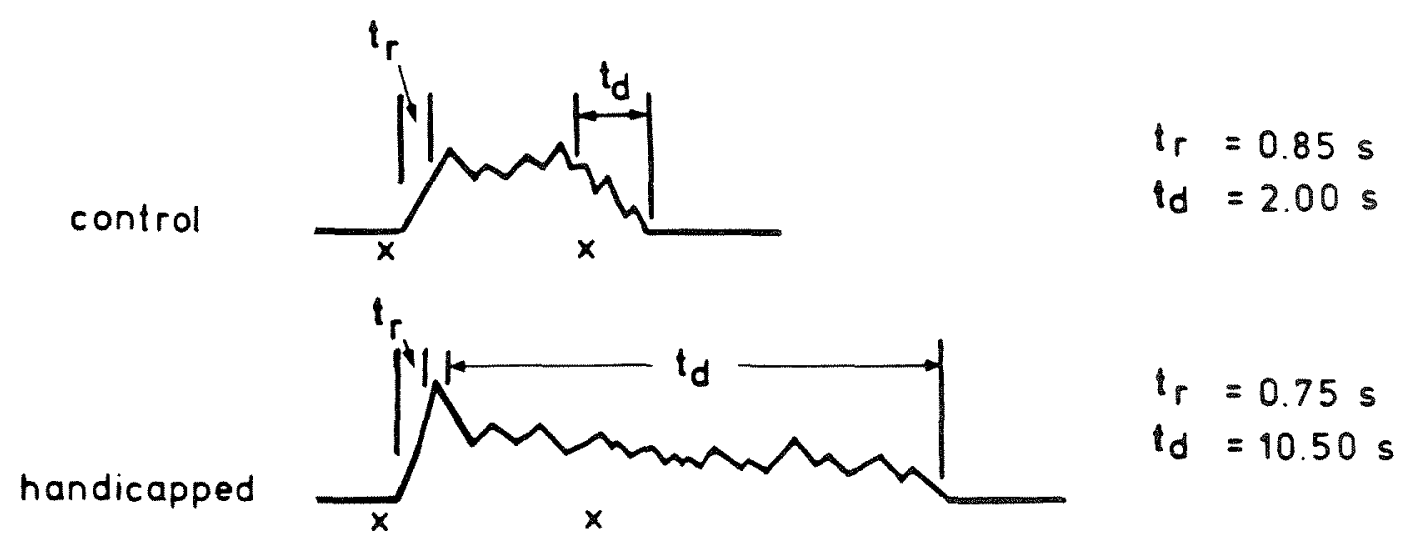

\section{FRONTALIS}
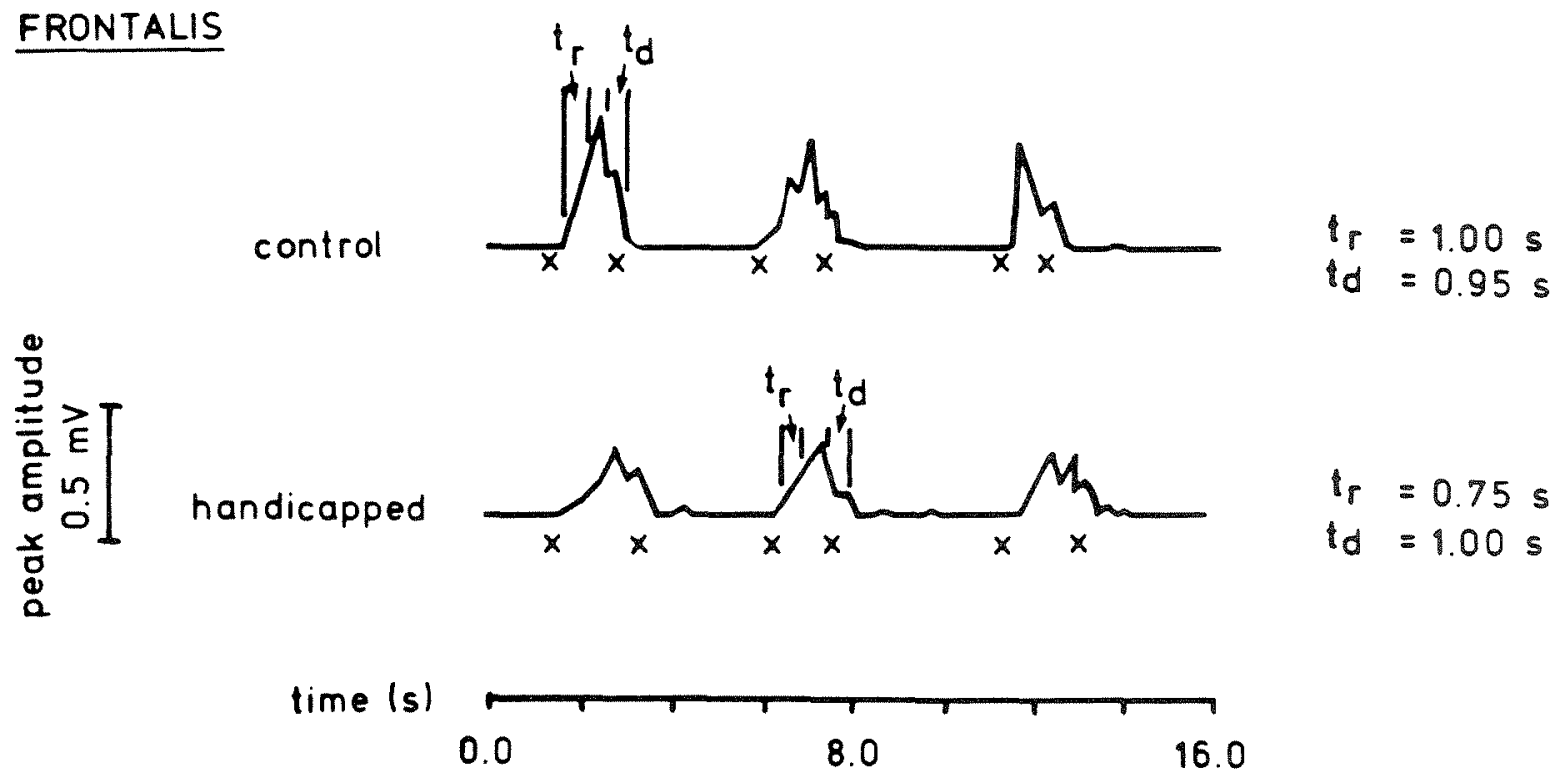

\section{Figure 8.}

Representative sampled peak amplitude envelopes, detailed ME evaluation. $\operatorname{tr}=$ rise time; td $=$ decay time; $\mathrm{x}=$ start or stop command. (top) Measured from left buccinator. Task: wide smile. (bottom) Measured from left side of frontalis. Task: full frontalis flexion. 
Table 2

Results of pursuit tracking task.

\begin{tabular}{|l|c|c|}
\hline task/subject & $\begin{array}{c}\text { maximum correlation } \\
\text { coefficient }^{*}\end{array}$ & $\begin{array}{c}\text { phase shift** } \\
\text { (degrees) }\end{array}$ \\
\hline $\begin{array}{l}\text { sine wave, 0.05 Hz } \\
\text { handicapped }\end{array}$ & 0.8436 & -0.71 \\
control 1 & 0.8778 & 0.76 \\
control 2 & 0.9471 & 0.24 \\
sine wave, 0.10 Hz & 0.6346 & -0.08 \\
handicapped & 0.7053 & 1.11 \\
control 1 & 0.9345 & 0.37 \\
control 2 & & \\
square wave, 0.10 Hz & 0.7332 & 2.32 \\
handicapped & 0.7882 & 1.53 \\
control 1 & 0.9289 & 1.95 \\
control 2 & \\
\hline
\end{tabular}

*calculated from one period (average of five periods).

$* *$ positive $=$ lag; negative $=$ lead

ingly positive. During the square-wave task, where the reference signal shifts rapidly from positive to negative extreme, no leading is observed. The handicapped subject's performance on this task (Figure 9, bottom) is notable for its slow response and total lack of overshoot; ignoring tremor, the curve resembles a low-pass-filtered version of the control response with a cutoff frequency of approximately 1.5 $\mathrm{Hz}$.

Several characteristic patterns were observed in the handicapped subject's performance during the evaluation. An oscillation of approximately $1 \mathrm{~Hz}$ frequency and 0.25-1.50 degrees of head rotation was observed throughout the evaluation. This pattern was labeled "Mode A."

The subject also exhibited two characteristic patterns of spastic movement, which emerged periodically during the trials. The first was characterized by a series of 1.0-2.0 Hz left-right oscillations over a 15-to-20-degree range. This pattern was labeled "Mode B." In the other pattern, "Mode C," the neck became rigid and the subject's head remained in a fixed position for 5 to 15 seconds. Mode B occurred once during each of the $0.10 \mathrm{~Hz}$ trials; Mode $\mathrm{C}$ occurred twice in the first trial and once in each of the others. (Waveform cycles which contained Mode B or C movement were not included in the data for cross-correlation.)

Despite these movement deficits and his slow response, the handicapped subject's tracking performance was still highly correlated with the reference signal (especially compared to control 1) and his deviations from the reference signal are regular and predictable. His pursuit tracking performance, while less precise than that of the normal subjects, indicates definite and potentially useful proportional control of side-to-side head rotation.

Multitarget Acquisition Task: Six average acquisition envelopes, each representing a common target-to-target path, were calculated for each subject. As in the tracking evaluation, characteristic differences were evident between the two control subjects, with one control demonstrating higher slopes and faster target acquisition in all cases. These differences consistently reached acceptable statistical significance for only one value, target entry slope (for one envelope, $\mathrm{p}=0.046$ ). Therefore, for statistical testing, data from the two controls were combined for all values except entry slope, for which each control value was tested separately against the handicapped subject's value.

The handicapped subject's mean time per target selection was significantly longer than that for either control, and his mean slopes at target exit and entry were significantly lower than the control values. (Figure 10) Neither time within target, nor time to reach zero slope after target entry, were consistently greater than control values, though significant differences in time within target were detected in two of the six average envelopes analyzed.

As in the tracking evaluation, abnormal movement patterns were observed in the handicapped subject's performance. The constant $1 \mathrm{~Hz}, 0.25-1.50$ degree oscillation (Mode A) was again observed, but only during movement to the right. Movement to the left was characterized by wider $1 \mathrm{~Hz}$ oscillations of 2 or more degrees amplitude, with the head returning to its original position approximately one second after initiation of the movement. These irregular oscillations account for the unusually high standard deviations of the parameters listed in Table 3. Spastic modes $\mathrm{B}$ and $\mathrm{C}$ were identical to those observed in the tracking evaluation, with Mode $B$ occurring twice during each trial and Mode $\mathrm{C}$ occurring once in one trial and twice in the other. (Only data recorded during Mode A were subjected to statistical analysis.)

\section{DISCUSSION}

The results of this case study demonstrate the usefulness of systematic motor assessment employing computer-aided evaluation techniques. Through 
SINE WAVE, $0.10 \mathrm{~Hz}$

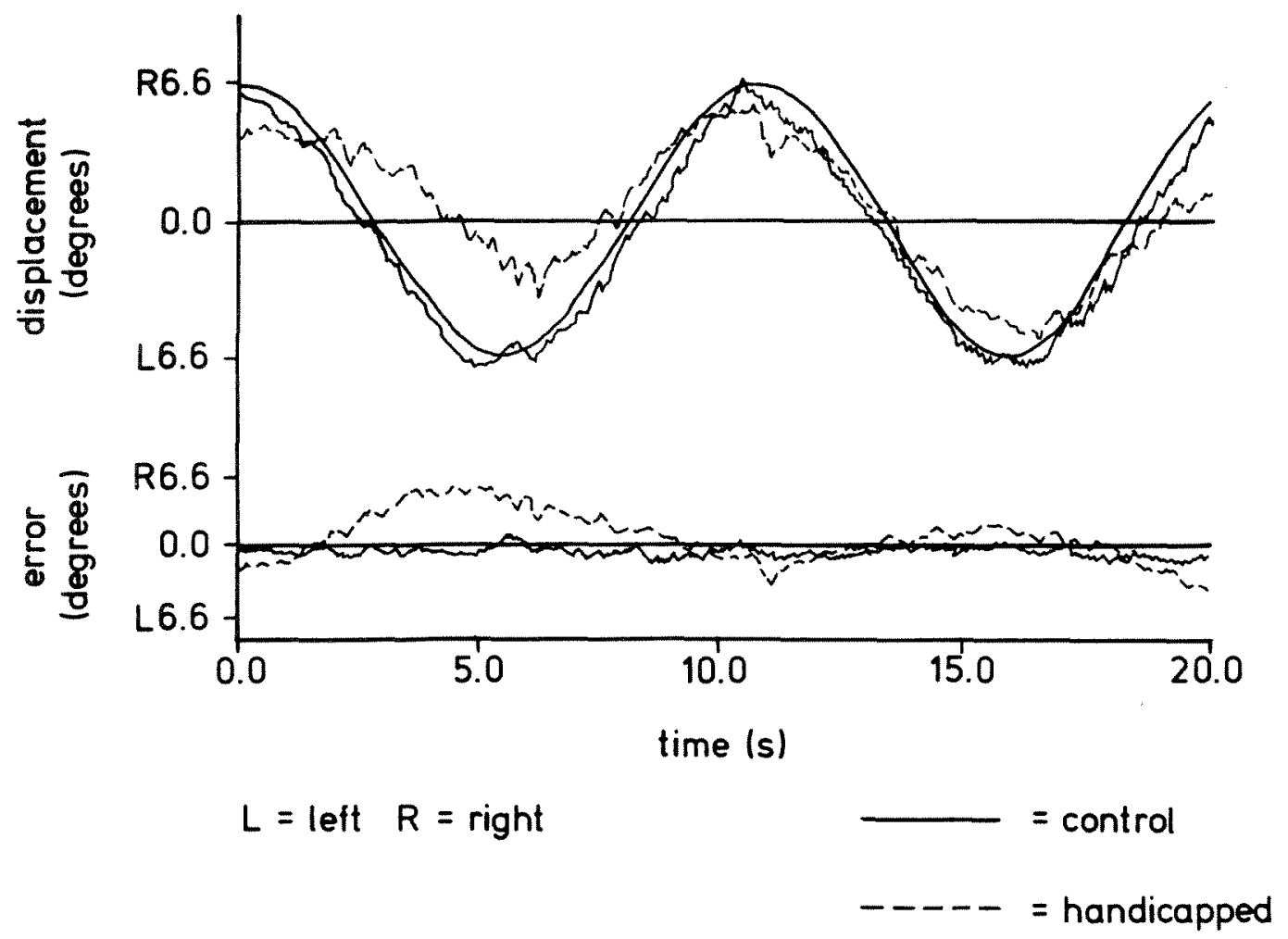

SQUARE WAVE, $0.10 \mathrm{~Hz}$

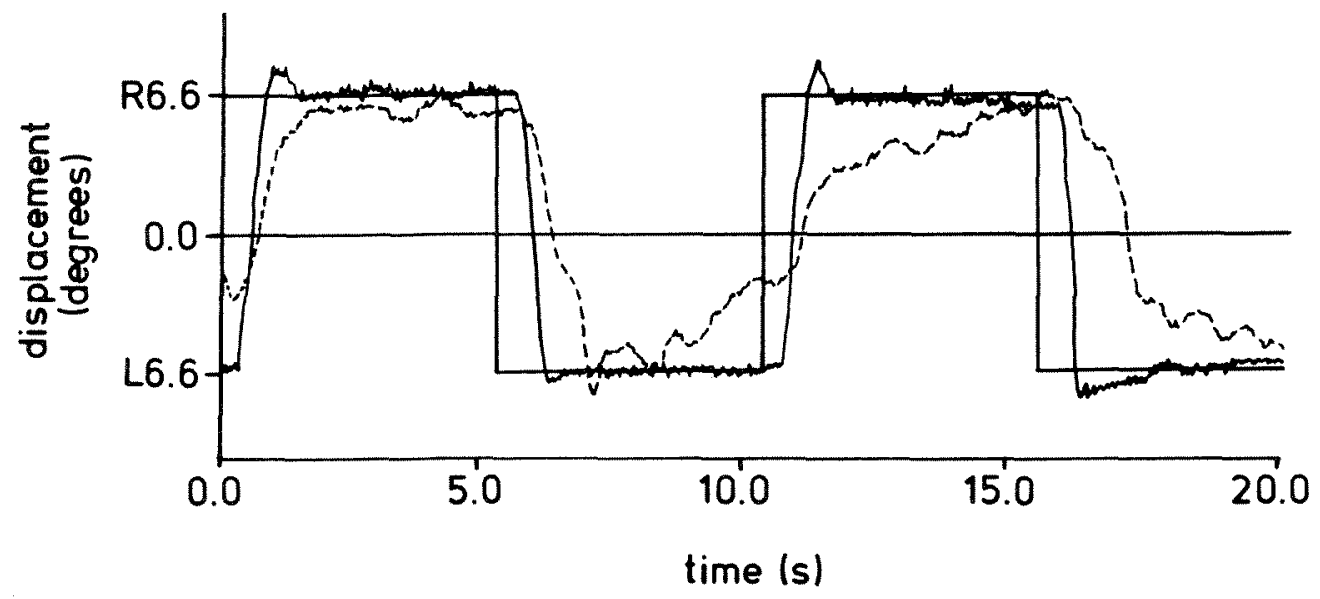

Figure 9.

Pursuit tracking task performance. Curves show left/right head displacement as compared to reference signal. Signals showing Modes B and C are not plotted. (top) Sine wave, $0.10 \mathrm{~Hz}$. Error curve shows deviation of response signal from reference signal. (bottom) Square wave, $0.10 \mathrm{~Hz}$. 
Table 3

Results of multitarget acquisition task. $* *=$ significant, $\mathrm{p}<.05$.

Multitarget Acquisition Task

\begin{tabular}{|c|c|c|}
\hline subject & $\begin{array}{c}\text { mean acquisition } \\
\text { time }(\mathrm{sec})\end{array}$ & statistical significance \\
\hline $\begin{array}{l}\text { handicapped } \\
\text { control } 1 \\
\text { control } 2\end{array}$ & $\begin{array}{l}3.733 \pm 4.166 \\
1.610 \pm 0.782 \\
1.354 \pm 0.540\end{array}$ & $\begin{array}{c}* * \text { handicapped } \times \text { control } 1: \\
t=15.37, \mathrm{p}=0.00 \\
* * \text { handicapped } \times \text { control } 2: \\
t=17.72, \mathrm{p}=0.00 \\
* * \text { control } 1 \times \text { control } 2: \\
t=2.57, \mathrm{p}=0.01\end{array}$ \\
\hline
\end{tabular}

\begin{tabular}{|c|c|}
\hline parameter/subject & statistical significance \\
\hline $\begin{array}{lc}\text { mean entry slope (degrees/sec) } \\
\text { handicapped } & 74.06 \pm 30.07 \\
\text { control } 1 & 114.37 \pm 40.54 \\
\text { control } 2 & 171.97 \pm 51.58\end{array}$ & $\begin{array}{c}* * \text { handicapped } \times \text { control } 1 \\
t=5.29, \mathrm{p}=0.00 \\
* * \text { handicapped } \times \text { control } 2 \\
t=11.67, \mathrm{p}=0.00 \\
* * \text { control } 1 \times \text { control } 2 \\
\mathrm{t}=6.72, \mathrm{p}=0.00\end{array}$ \\
\hline $\begin{array}{lr}\text { mean exit slope }(\text { degrees/sec) } \\
\text { handicapped } & 93.76+48.98 \\
\text { controls } & 183.76 \pm 74.05\end{array}$ & $\begin{array}{c}* * \text { handicapped } \times \text { controls } \\
\mathrm{t}=7.95, \mathrm{p}=0.00\end{array}$ \\
\hline
\end{tabular}

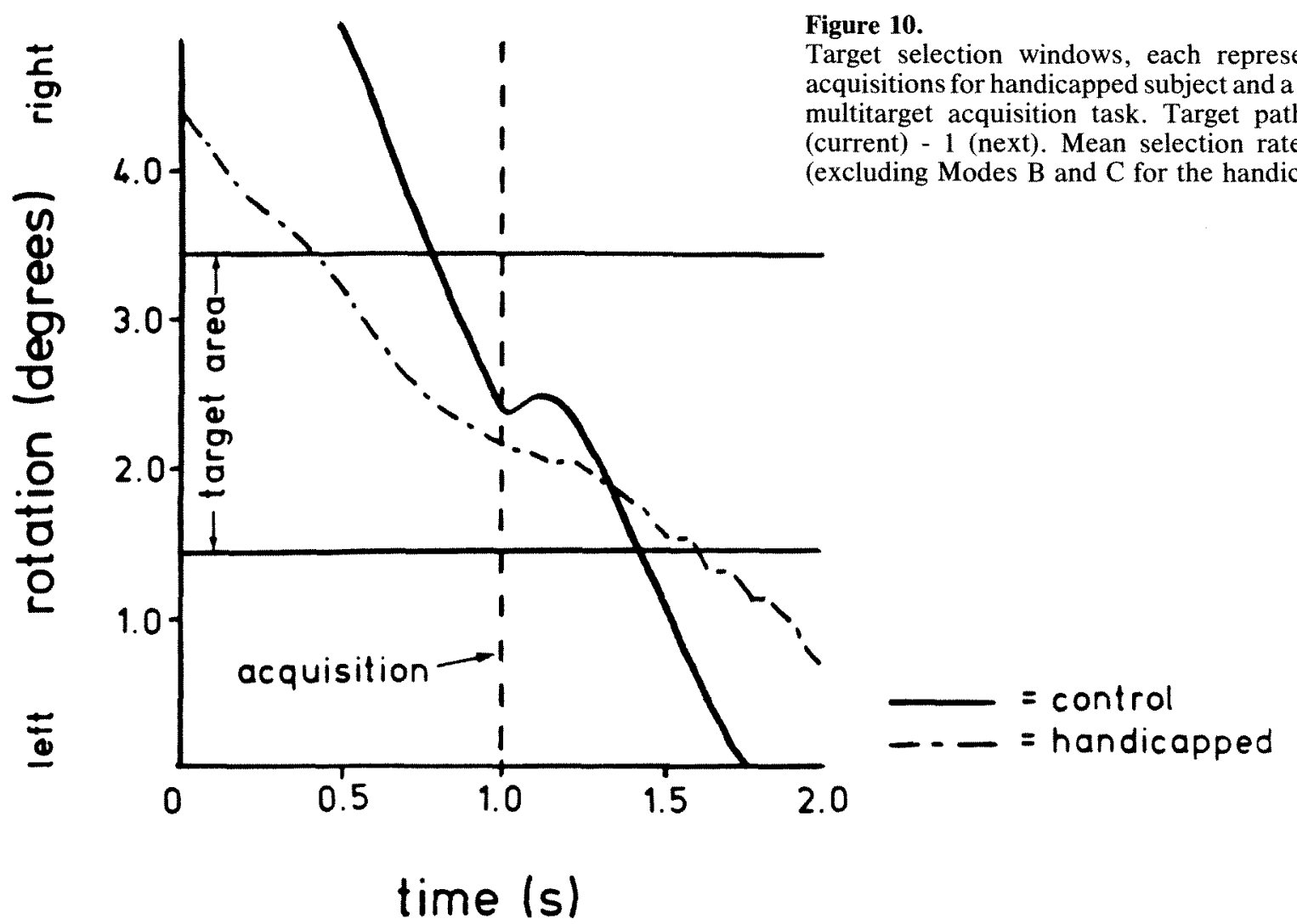

quantification of the handicapped subject's volitional myoelectric activity, his control of the frontalis was discovered to be suitable for communication device operation. The displacement evaluation revealed several characteristic deficits in the handicapped subject's proportional control of head rotation. These abnormalities do not, however, render head rotation unusable as a control modality. Mode A slowed but did not fully impair the subject's ability to track a reference signal or select from an array of targets; Modes $\mathrm{B}$ and $\mathrm{C}$ are easily recognized and categorized. Given these results, if steps are taken to compensate for these deficits, the subject will possess usable proportional control exceeding that necessary for operation of his current UNICOM system. Some possible compensatory measures are addressed later in this section.

The myoelectric evaluation identified an ME signal site which could potentially be used for device control. Signals of varying amplitude from a single site can be used to proportionally control devices such as myoelectric prostheses (22). However, though

Target selection windows, each representing average of 12 a control subject during path is 3 (previous) - 2 (current) - 1 (next). Mean selection rates are for entire trial excluding Modes $\mathrm{B}$ and $\mathrm{C}$ for the handicapped subject). 
Journal of Rehabilitation Research and Development Vol. 24 No. 3 Summer 1987

ME signals from the subject's frontalis have nearnormal parameters, they are not proportionally controlled. Therefore, they are useful only for binary switching, either "on" or "off" (20). Used alone, these signals would have no clear advantage over the subject's current head switch as a method of device control. However, they may be effective if used in combination with proportional head rotation, or in addition to the subject's current head switch. The displacement evaluation revealed that the subject could use head rotation to directly control the movement of cursors projected on a computer screen, and to select from an array of displayed items at a rate substantially exceeding the UNICOM selection rate (16 as opposed to 7.5 selections per minute). This mode of device control is known as direct selection (16). The major advantage of proportionally-controlled direct selection over the subject's current scanning method is that the user is free to skip over unwanted items and proceed directly to the desired language unit, while scanning requires that the user wait for the device to scroll through the entire list of unwanted items before the desired item can be chosen (4). A direct-selection device would therefore appear to be desirable for this handicapped subject. The subject's proportional head movement can be translated into device control-voltages using either modified joysticks (11), ultrasonic detectors (8), electromagnetic systems such as the one used during the evaluation, or numerous other interfaces. To further increase the communication rate, myoelectric signals from the frontalis could be used to trigger item selection instead of the delay-time system used during the multitarget acquisition task.

The utilization of proportional head movement to control communication or other assistive devices is complicated by the handicapped subject's characteristic movement deficits. However, given the availability of quantitative descriptions of each of these deficits, it may be possible to adjust the hardware interface between user and device to compensate for them. For example, a proportional control interface that restrains the head and provides slight resistance to movement may minimize the subject's characteristic low-amplitude oscillations (Mode A). Alternately, electronic adjustment of the individual's control output, either in hardware or in software, could transform it to resemble the signals produced by nonhandicapped persons. Neither of these meas- ures may be sufficient to compensate for the handicapped subject's periodic shifts into spastic oscillations (Mode $\mathrm{B}$ ) or rigidity (Mode $\mathrm{C}$ ), which render him temporarily unable to control his head motion. However, this problem can be solved by specialized programming of the communication aid itself, because the assessment results demonstrate that Modes $\mathrm{B}$ and $\mathrm{C}$ are characterized by distinct movement patterns that can be represented as mathematical functions. A communication aid could be programmed to "recognize" the occurrence of those functions and to temporarily suppress aid operation whenever they occur. Control would be restored to the user after the abnormal movements ceased. As artificial intelligence technology progresses, this type of device customization will become increasingly feasible.

This last application underscores the potential versatility and effectiveness of the above-described procedures as clinical tools. Their high level of precision and nonsubjective nature increase the likelihood of detection of "hidden" control sites. Quantitative data are universally understood and easily transmitted among professionals and institutions. Their usefulness can extend outside the communication clinic because comprehensive profiles of patient movement may be of use to physical therapists, physicians, orthopedic specialists, and other health professionals as well as the engineers who design prosthetic and orthotic devices. However, a substantial database of normal subjects must be collected before these procedures can fulfill their potential. In the present study, the comparisons of nonhandicapped with handicapped subjects would have been better supported with a larger number of control subjects. In addition to gathering a larger database, the authors intend to investigate the issue of varying cognitive abilities in severely handicapped individuals. It is hoped that additional quantitative data on these groups will strengthen statistical support of our procedures' results and add to our understanding of the nature of motor deficits in the severely handicapped.

Another difficulty lies in the area of device prescription. Quantitative descriptions of motor performance cannot be readily applied to the prescription of particular devices in the absence of numerical specifications for those devices. The performance parameters of devices currently on the market have not been collected in any organized fashion, though 
major efforts to do so are now under way (6). The creation of this database will markedly increase the utility of quantitative performance data in the patient/device matching process.

In the present study, the scope of focused assessment was necessarily limited to the specific capabilities of the handicapped subject. The generality of the assessment protocols must be increased if they are to deal satisfactorily with a wide range of motor deficits. Further research now in progress focuses on the expansion of these protocols using instrumentation available at the NeuroMuscular Research Center. Displacement, its time derivatives, and axes of rotation can be computed and displayed using a computer-based system incorporating infrared-detection motion analysis hardware (WATSMART) (21) and sophisticated kinematic analysis software (TRACK) (1). This system, unlike the motion transducer used in the present study, assesses motion in three dimensions and can simultaneously examine and compare movement at up to 12 body sites at resolutions as high as $1 \mathrm{~mm}$, at sampling rates ranging well over $100 \mathrm{~Hz}$. Virtually any motor deficit can be evaluated and quantified using this powerful technique. A series of tests is now underway using the system concurrently with the techniques reported in this study.

Another measure under consideration is the objective quantification of muscle fatigue using the Muscle Fatigue Monitor (MFM), a device that measures changes in the median frequency of $\mathrm{ME}$ signals (5). Fatigue rate is an important consideration when evaluating a muscle's suitability for use in device control, as such control must often be exercised over long periods of time. The MFM provides clear quantitative fatigue parameters that can be compared with those for normal subjects. Studies are

\section{REFERENCES}

1. Antonsson EK: A Three-Dimensional Kinematic Acquisition and Intersegmental Dynamic Analysis System for Human Motion. Ph.D. Thesis in Mechanical Engineering, Massachusetts Institute of Technology, 1982.

2. Basacchi A, Naumann S, Mrlner M: Microprocessorbased assessment of controller interfaces for disabled users. now being designed to evaluate the effectiveness of MFM evaluation in the motor assessment process. The eventual goal is the development of an integrated, microcomputer-driven motion analysis system using the WATSMART hardware as a nucleus. Its extensive data storage capabilities and capacity to accept inputs from external sources will allow the simultaneous operation of WATSMART, TRACK, the MFM, and the techniques used in the present study. Such a system would produce a comprehensive profile of any subject's motor abilities, with displacement, myoelectric, and fatigue parameters for each potential device-control site as well as data on the interaction of those sites.

These quantitative techniques, while they hold great promise, are not meant to replace the clinical measures currently in use, but rather to supplement the clinician's own skills and observations. It must be remembered that the final decision as to an appropriate assistive device must be based on the patient's particular needs and desires as much as on his or her motor abilities. But with the development of an integrated, quantitative assessment system and an expanded subject database, the clinician's ability to evaluate patients and prescribe the most suitable assistive devices will be markedly increased.

\section{Acknowledgments}

The authors are deeply grateful to Richard Hoyt, the disabled subject, and his family and personal care staff for being such willing participants and for providing the inspiration for this effort. We also thank the following individuals for their invaluable assistance and support: Leonard Saxe; Leonard Zaichkowsky; Larry Andrus; Kurukundi Murthy; Zvi Ladin; Howard Shane; Michelina Cassella; Michael Rosen; Cheryl Goodenough-Trepagnier; and Claire McCarthy.
Proceedings of the Fourth Annual Conference on Rehabilitation Engineering, 295-297, 1981.

3. Coleman CL, CoOK AM, Meyers LS: Assessing non-oral clients for assistive communication devices. I Speech Hear Disorders45: 515-526, 1980.

4. GADDIS EL: Proportional computer interface increases 
speed of nonvocal communication. Proceedings of the IEEE Computer Workshop on Computing to Aid the Handicapped, 69-75, 1982.

5. GILmore LD, AND DE LuCA CJ: Muscle fatigue monitor (MFM): Second generation. IEEE Transactions on Biomedical Engineering 32:1, 75-78, 1985.

6. Goodenough-Trepagnier C And Rosen MJ: A prescriptive system for selecting communication devices for nonspeaking patients. Interim report, April 1984.

7. Hacisalihzade SS, Kuster F, Albani C: Computeraided measuring of motor functions using pursuit tracking. Computer Methods and Programs in Biomedicine 23, 1928, 1986.

8. JAFFE DL: An ultrasonic head position interface for wheelchair control. Proceedings of Johns Hopkins First National Search for Applications of Personal Computing to Aid the Handicapped, 142-145, 1981.

9. JANDURA L: Fitts Law Modeling of a 2-Degree of Freedom Video Tapping Task Using a Displacement Joystick as Input. Unpublished paper, Massachusetts Institute of Technology, 1985.

10. JonES RD AND DONALDSON IM: Measurement of sensorymotor integrated function in neurological disorders: Three computerized tracking tasks. Med Biol Eng Comput 24, 536-540, 1986.

11. Lozac'h Y, Gossalin G, Sherman ED, Gingras G, RITCHUK M: New head control for quadriplegic patients. Bul Prosthet Res 10-23, 151-157, 1975.

12. MCCARTHY CF AND DE LuCA CJ: A myofeedback instrument for clinical use. Bull Prosthet Res 10-40, 39-44, 1984.

13. Pheasant S: Bodyspace: Anthropometry, Ergonomics and Design. Philadelphia: Taylor and Francis, 1986.
14. Prentke-Romich Company: All Communications Aids Are Not Created Equal. (Educational service flyer) 1985.

15. REMMEL RS: An inexpensive eye movement monitor using the scleral search coil technique. IEEE Transactions on Biomedical Engineering 31:4, 388-390, 1984.

16. Rosen MJ and Goodenough-Trepagnier C: Communication systems for the nonvocal motor handicapped: Practice and prospects. Eng Med Biol, 31-35, December 1982.

17. Rosen MJ, Goodenough-Trepagnier C, Miller J, DalRYMPle G, Jandura L, Getschow C, Leung S, Lee S: Computer-aided motor assessment and rate prediction for prescription of communication devices. Proceedings of the RESNA 8th Annual Conference, Memphis, Tennessee, 326$328,1985$.

18. Rowell D AND DALRYMPLE GF: The UNICOM: A microprocessor-based communicator for the nonvocal severely motor impaired. Proceedings of Johns Hopkins First National Search for Applications of Personal Computing to Aid the Handicapped, 152-155, 1981.

19. Rudin NJ: A Motor Assessment Procedure for Communication Device Prescription. Unpublished B.A. Thesis, Boston University College of Liberal Arts and NeuroMuscular Research Center, 1986.

20. UPJOHN LR: A wireless remote control for microcomputer systems using voluntary electromyographic activity as the controlling signal. Proceedings of Johns Hopkins First National Search for Applications of Personal Computing to Aid the Handicapped, 181-182, 1981.

21. WATSMART: Waterloo Spatial Motion Analysis and Recording Technique. Northern Digital Inc., 403 Albert Street, Waterloo, Ontario N2L 3V2, Canada.

22. Williams TW: The Boston Elbow: The path to a mature myoelectric prosthesis. Soma Magazine, 30-33, July 1986. 